\title{
Sobre la función narrativa del pretérito anterior*
}

\author{
Guillermo Soto** \\ Universidad de Chile
}

\begin{abstract}
Resumen
Se explora la función narrativa del pretérito anterior (hubo llegado, PA) en pasajes de El Quijote de la Mancha. El estudio muestra que, en el corpus analizado, el PA se emplea fundamentalmente en cláusulas subordinadas temporales que introducen logros vendlerianos en narraciones. Si bien estos logros se sitúan temporalmente en la línea del primer plano narrativo, el PA los subordina a la situación designada en la cláusula principal, tratándolos como parte del segundo plano narrativo. En los casos en que opera sobre situaciones no puntuales (estados, actividades o realizaciones vendlerianas), el PA desencadena una coerción aspectual a logro. El PA comunica eventos de gran dinamismo que evocan rapidez, violencia o emoción.
\end{abstract}

Palabras clave: pretérito anterior, sistema verbal del español, narración, aspecto, coerción aspectual.

* La preparación de este trabajo contó con el apoyo de los proyectos Fondecyt 1070935 y 1110525 . Una versión muy preliminar se expuso en el Tercer Seminario Nuevas Lecturas en Torno al Quijote, Santiago, Universidad de Chile, 5 de noviembre de 2008.

** Para correspondencia, dirigirse a: Guillermo Soto (gsoto@uchile.cl), Departamento de Lingüística y Centro de Estudios Cognitivos, Facultad de Filosofía y Humanidades, Universidad de Chile, Ignacio Carrera Pinto 1025, Tercer piso, Nuñoa, Santiago, Chile. 


\title{
ON NARRATIVE FUNCTION OF SPANISH PAST ANTERIOR
}

\begin{abstract}
This work explores the narrative function of Spanish past anterior (hubo llegado, PA) in passages taken from Don Quixote. The study shows that, in this corpus, PA is typically used in subordinate temporal clauses that introduce vendlerian achievements in narratives. Although these achievements are part of the temporal foreground of the narrative, PA subordinates them to a main clause, treating them as part of narrative background. When PA operates on a non punctual situation (vendlerian states, activities or accomplishments), it triggers an aspectual coercion to achievement. PA comunicates very dynamic events that evoke speed, violence or emotion.
\end{abstract}

Key words: past anterior, Spanish verbal system, narrative, aspect, aspectual coercion.

Recibido: 03/02/13 Aceptado: 03/05/13

\section{INTRODUCCIÓN}

El pretérito anterior (hube hecho), en adelante PA, es una forma verbal obsoleta en español, aunque no caduca (Octavio de Toledo y Rodríguez 2008; García 2008; Soto 2009). La bibliografía especializada concuerda en que su empleo, desde hace ya tiempo, se haya restringido a la lengua escrita ${ }^{1}$, y aun en esta es muy escaso y llega a connotar arcaísmo y afectación. En otro estudio, destinado a una caracterización semántico-pragmática de la construcción, hemos encontrado en Chile usos en los siglos XVI y XVII y a partir de 1845 hasta nuestros días (Soto 2009). En los usos contemporáneos, son importantes los escritores de prestigio; no obstante, el empleo no se restringe a textos históricos y literarios, pues también hay casos esporádicos en el ámbito periodístico.

1 Miranda (1980-1981) refiere un caso en un corpus oral de norma estándar de Santiago (Chile), correspondiente al $0,01 \%$ de todas las formas verbales finitas y no finitas de su muestra. Lamentablemente, no presenta la cláusula en cuestión. Bull (1947), por otro lado, en su estudio de textos literarios narrativos, líricos, dramáticos y ensayísticos, no encuentra casos. Criado de Val (1948), por su parte, encuentra muy pocos casos en obras dramáticas de Benavente (cfr. Moreno de Alba 2006). 
La modalidad narrativa favorece considerablemente la presencia de PA (Hurtado 2001; Soto 2009), lo que indica que esta estructura desempeña alguna función en ella. Por otra parte, la persistencia de casos de PA en autores literarios de prestigio, aunque muy esporádica, sugiere que estos son capaces de distinguir dicha función de la desempeñada por construcciones verbales relacionadas, como el pluscuamperfecto. El presente trabajo tiene por objeto caracterizar el funcionamiento del PA en el discurso narrativo literario, observando, especialmente, su papel en pasajes de El Quijote de la $M a n c h a^{2}$. En primer término, se caracteriza sintéticamente la construcción, de acuerdo con Soto $(2009)^{3}$, relevando los rasgos aspectuales pertinentes para su función discursiva. Posteriormente, se buscan los casos de PA presentes en la obra y se analiza el papel de la construcción en la organización temporal e informativa de los pasajes narrativos en que figura. De acuerdo con el análisis propuesto, el PA se emplea narrativamente en cláusulas temporales subordinadas y opera como un introductor de eventos puntuales, o logros vendlerianos (Vendler 1967). Si bien los logros introducidos se sitúan en la línea temporal del primer plano narrativo, el PA explicita su subordinación a la situación designada en la cláusula principal, respecto de la cual sirven, mayoritariamente, como punto de arranque. Cuando opera sobre situaciones que no son logro, el PA desencadena coerción aspectual a logro. En los casos observados, el PA comunica eventos altamente dinámicos y evoca rapidez, violencia o emoción. Estas funciones contrastan con las del pretérito pluscuamperfecto, que típicamente introduce estados que forman parte del segundo plano narrativo. La propuesta se ilustra con casos seleccionados de El Quijote.

\section{EL PRETÉRITO ANTERIOR}

Ya en el Medioevo, el empleo del PA es muy minoritario, al menos desde el siglo XII, de acuerdo con Hurtado (2001) y Moreno de Alba (2006). Las frecuencias que registra este último autor indican que, del conjunto de formas verbales de pasado, en El Cid (fines del siglo XII, principios del XIII), el PA

\footnotetext{
2 Se emplea la edición crítica de 1998 de Francisco Rico, accesible en el Centro Virtual Cervantes. URL: http://cvc.cervantes.es/literatura/clasicos/quijote/default.htm [consulta $20 \mathrm{de}$ diciembre de 2012].

3 Una caracterización semejante, aunque desde otra perspectiva teórica, en García (2008).
} 
alcanzaba el 0,5\%; en la General Estoria de Alfonso X, fines del siglo XIII, el 0,2\%; y en la Crónica del Rey don Pedro, de Pero López de Ayala, siglo XV, el 0,3\%. Sube en La Celestina, siglo XVI, al 0,6\%. Y en El Quijote, inicios del siglo XVII, alcanza el 0,3\%. Para aquilatar adecuadamente estos porcentajes, valga la comparación con el pluscuamperfecto, que, siguiendo con las cifras de Moreno de Alba, es porcentualmente el doble en $\mathrm{El} \mathrm{Cid}$ $(1,0 \%)$; mucho más del doble en la General Estoria (4,5\%), aunque en este caso considerando tanto el pluscuamperfecto analítico con haber como el sintético en -ara, esto es, llegara en su uso no de imperfecto del subjuntivo sino de pluscuamperfecto del indicativo, de acuerdo con su valor etimológico. En La Celestina, el porcentaje del pluscuamperfecto alcanza el 3,0\%, también con algunos pluscuamperfectos del tipo llegara, y en El Quijote, el 4\%.

La comparación de los datos aportados por Moreno de Alba es de interés porque, al menos desde Bello (1841/1972), se viene afirmando que el PA es conmutable por el pluscuamperfecto y que ambos comunican una situación anterior a otra situación pasada respecto del momento de enunciación. Así, podemos decir:

(1) Apenas hubo terminado de cenar se levantó de la mesa.

(2) Apenas había terminado de cenar se levantó de la mesa.

La diferencia entre estos dos pretéritos compuestos sería tan solo que mientras el anterior especifica anterioridad inmediata respecto de la situación pasada, el pluscuamperfecto no lo hace. Sin embargo, al emplearse un elemento que explicita una relación de inmediatez, como apenas, en los ejemplos expuestos, dicha diferencia desaparecería. En consecuencia, la mayor parte de los autores sostiene que ambas construcciones son funcionalmente equivalentes en sus empleos de anterioridad inmediata en español. Esta sería, según se dice, la causa de la obsolescencia del PA, que vendría siendo desplazado por el pluscuamperfecto, y aun por el pretérito indefinido ${ }^{4}$, desde hace siglos en español.

A pesar de tratarse de una postura bastante generalizada, el análisis sintetizado presenta serias limitaciones descriptivas, toda vez que las condiciones de empleo del PA van más allá de su valor como tiempo relativo a otro tiempo (García 2008; Soto 2009). En efecto, el PA parece exigir que la situación sobre la que opera sea dinámica y télica, esto es, que tenga

4 Las gramáticas académicas vienen llamando "pretérito perfecto simple" a canté (RAE 1973; RAE 2009); acá se emplea la expresión "pretérito indefinido" para evitar su asociación con la categoría semántico-gramatical de perfecto (he cantado). 
despliegue de energía y sea discreto. Ninguna de estas dos condiciones es aplicable al pretérito pluscuamperfecto, que se utiliza tanto con eventos dinámicos como con estados y tanto con eventos télicos como con eventos atélicos. Estas diferencias pueden ilustrarse en el contraste entre la cláusula estativa (3) y la (4), agramatical o, al menos, muy extraña.

(3) Se incendió el quincho donde un día antes había estado Guillermo Moreno 5 .

(4) *Se incendió el quincho donde un día antes hubo estado Guillermo Moreno.

Ligado a esto, y más interesante aun en relación con el presente trabajo, hemos sostenido que el PA focaliza el instante de cambio o transición de una situación dinámica y télica - un logro, en el sentido de Vendler (1967)-; es decir, el momento en que se inicia un estado resultativo. Así, la oración

(5) $[\mathrm{Y}]$ no hubo tocado al suelo, cuando se levantó más ligero que un gamo (El Quijote, $1^{\mathrm{a}}$ parte, cap. XXI),

se focaliza en el instante preciso en que don Quijote toca el suelo y empieza inmediatamente a levantarse. Como se observa, la construcción muestra un gran despliegue de energía, manifiesto en el empleo del se medio dinamizador (Maldonado 1999, Soto y Muñoz 1999-2000) y la comparativa más ligero que un gamo.

Mientras el pluscuamperfecto opera, al menos en uno de sus usos narrativos, como un estativizador que genera un estado resultante, el PA comunica, en el análisis que proponemos, un evento puntual télico (un logro) que, canónicamente, marca el límite inicial de otro evento (Soto 2009). Esto significa que, a diferencia del pluscuamperfecto, el anterior introduce una entidad discreta en el discurso; típicamente, en el narrativo.

En los casos en que el PA opera sobre una situación que no es logro (esto es, un estado, una actividad o una realización vendleriana), desencadena una coerción aspectual que la puntualiza, transformándola en un logro vendleriano. Esto, pensamos, obedece a una reinterpretación contextual del significado aspectual del verbo léxico no puntual, desencadenada por la incompatibilidad aspectual entre la unidad léxica y el PA (para una

\footnotetext{
Tomado de http://www.taringa.net/posts/noticias/2279251/Moreno,-queman-quinchoen-donde-habia-estado_.html [Consulta, 31 de agosto de 2009].
} 
caracterización detallada de la coerción, cfr. Francis y Michaelis 2003). En la sección 4, se desarrolla e ilustra el fenómeno.

\section{EL PAPEL DE LA ASPECTUALIDAD EN EL PRIMER PLANO NARRATIVO}

Tradicionalmente, el criterio para reconocer como narrativo un discurso ha sido la existencia de, al menos, una secuencia de dos cláusulas temporalmente ordenadas, denominadas cláusulas narrativas (Labov y Waletzky 1967; Reinhart 1984). En estas, su orden formal replica el orden en que se suceden los eventos en el mundo narrado. Así, la secuencia:

(6) María fue al mercado y compró un dulce

está conformada por dos cláusulas narrativas, en tanto los dos eventos se presentan en el mismo orden en que ocurren en el mundo ${ }^{6}$. En contraste, las cláusulas de la secuencia

(7) María fue al mercado una vez que volvió del doctor

no constituyen una secuencia narrativa, pues el orden de aparición de las cláusulas no se corresponde con el orden en que ocurren los eventos. Por supuesto, los textos narrativos típicamente contienen muchas cláusulas no narrativas. La restricción expuesta solo exige que haya al menos una secuencia narrativa, esto es, dos cláusulas narrativas en un texto, para que este cuente como narración. La secuencia de cláusulas narrativas establece el esqueleto de la narración, su eje temporal, denominado primer plano o prominencia. Como señala Reinhart (1984), el componente no narrativo se organiza alrededor del esqueleto narrativo y aporta los detalles que permiten reconstruir el mundo representado y establecer el significado y propósito del texto. El material no narrativo se denomina segundo plano o trasfondo. Así, en un enunciado como

(8) Andrea se fue Buenos Aires porque quería especializarse en diseño gráfico. Allí se hizo amiga de Isolda

6 Por supuesto, 'mundo' no debe entenderse necesariamente como el mundo real. 
las cláusulas Andrea se fue a Buenos Aires y allí se hizo amiga de Isolda están en primer plano, mientras que la cláusula quería especializarse en diseño gráfico está en trasfondo y, en este caso, especifica la motivación del evento de irse a Buenos Aires. Es importante precisar que esta distinción, al menos como la entiende Reinhart, no dice relación con la importancia o relevancia de los segmentos. Con frecuencia, lo más importante de los relatos está en el trasfondo ${ }^{7}$. Como se entiende en el presente trabajo, tampoco se corresponde directamente con opciones gramaticales específicas, como en Labov (1997), para quien la subordinación implica segundo plano ${ }^{8}$. La función de la dicotomía es resolver el problema de la ubicación temporal de los eventos en los relatos: la prominencia es la secuencia de cláusulas que mueven hacia adelante la línea temporal de la historia. Cada cláusula narrativa hace avanzar el punto de referencia temporal que permite ubicar el evento referido por la cláusula siguiente. Así, en el ejemplo (8), la primera cláusula narrativa (Andrea se fue Buenos Aires) hace avanzar el punto de referencia temporal, y respecto de ese nuevo punto se ubica el hacerse amiga con Isolda. Esto quiere decir que se hace amiga de Isolda después de haberse ido a Buenos Aires.

Como se explica más detalladamente en Soto (2011), un requisito para que esto ocurra es que las cláusulas narrativas refieran a eventos télicos, es decir, limitados, y no a estados. Esto, porque los estados, por su carácter ilimitado, trascienden los tiempos de referencia. Así, en el ejemplo, el querer especializarse en diseño gráfico es un estado vigente al momento de partir a Buenos Aires, pero eventualmente también cuando Andrea se hace amiga de Isolda y aun después de ello. Considerando la homología entre el dominio de

\footnotetext{
La posición sostenida por Reinhart y adoptada en el presente trabajo contrasta con la de quienes, como Hopper y Thompson (1980) y Unger (2002), asignan al primer plano una preeminencia informativa. Para Reinhart, el primer plano obedece a una proyección del dominio de la percepción espacial al campo del lenguaje tal que primer plano corresponde a figura y segundo plano a fondo, y no implica, consecuentemente, mayor importancia o relevancia de la información comunicada. A pesar de proponerse una homología entre la dicotomía figura/ fondo espacial y la dicotomía prominencia/trasfondo lingüística, la distinción de Reinhart tampoco debe interpretarse en el sentido propuesto por Talmy (2000) para la dicotomía figura/ fondo en el lenguaje. El tema se trata más detenidamente en Soto (2011).

8 Por supuesto, esto no significa que no haya relación entre las opciones gramaticales y el plano narrativo. Como han expuesto Hopper y Thompson (1980), rasgos asociados a la estructura argumental, la morfología gramatical o el empleo de ciertos marcadores morfológicos se correlacionan con un plano u otro (en el mismo sentido, Reinhart 1984). El punto es que el plano se determina a través de criterios distintos de los puramente morfosintácticos. Esto permite dar cuenta de discordancias entre la marcación gramatical de una cláusula y la secuencia temporal del discurso, como en El niño se tomó de la rama que se quebró y se cayó (cfr. Bocaz 1997).
} 
las entidades y el de las situaciones (Talmy 2000), lo anterior viene a querer decir que las cláusulas que introducen individuos pueden formar parte del primer plano narrativo, mientras que las que introducen masas, no.

\section{EL PRETÉRITO ANTERIOR Y LOS PLANOS NARRATIVOS}

A partir de lo expuesto, es posible conectar la dicotomía temporal entre prominencia y trasfondo narrativos con la distinción entre pluscuamperfecto y PA. El pluscuamperfecto puede entenderse, típicamente, como un recurso para poner en trasfondo cierta información, con independencia de su estatus intrínseco, una función que ya destacaran Reinhart (1984) y Mackenzie (1999). Esta función se observa en ejemplos como los siguientes, tomados de El Quijote:

(9) y sin que nadie le viese, una mañana, antes del día, que era uno de los calurosos del mes de julio, se armó de todas sus armas, subió sobre Rocinante, puesta su mal compuesta celada, embrazó su adarga, tomó su lanza, y, por la puerta falsa de un corral, salió al campo con grandísimo contento y alborozo de ver con cuánta facilidad había dado principio a su buen deseo ( $1^{\mathrm{a}}$ parte, cap. II).

(10) Apenas había el rubicundo Apolo tendido por la faz de la ancha y espaciosa tierra las doradas hebras de sus hermosos cabellos, y apenas los pequeños y pintados pajarillos con sus harpadas lenguas habían saludado con dulce y meliflua armonía la venida de la rosada aurora, que, dejando la blanda cama del celoso marido, por las puertas y balcones del manchego horizonte a los mortales se mostraba, cuando el famoso caballero don Quijote de la Mancha, dejando las ociosas plumas, subió sobre su famoso caballo Rocinante ( $1^{\text {a }}$ parte, cap. II).

En (9), el pluscuamperfecto no hace avanzar temporalmente el relato, conservando el punto de referencia temporal de la cláusula precedente, respecto del cual establece una relación de anterioridad. La narración se ubica en el momento posterior al principio del buen deseo de don Quijote, que es cuando este siente el grandísimo contento. Obsérvese que, en (10), la relación entre el despuntar del alba y el canto de los pájaros, de un lado, y el 
montarse a caballo, del otro, es de anterioridad inmediata. No obstante, las primeras cláusulas, que construyen el trasfondo del evento prominente -el subirse de don Quijote a su caballo- van en pluscuamperfecto. Esta función es esperable en tanto el pluscuamperfecto puede ser, como ya se ha dicho, un estativizador, esto es, una construcción que introduce una masa en el discurso.

El PA, en cambio, por introducir individuos en el discurso, permite comunicar información que hace avanzar temporalmente el relato. Esta información se conecta con la aportada por una cláusula en pretérito indefinido, que es, de acuerdo con la bibliografía, el tiempo típico del primer plano. En otras palabras, la hipótesis que se sostiene en este trabajo es que en los usos del PA en El Quijote, el autor opta por esta construcción, ciertamente facultativa, para comunicar dos eventos íntimamente conectados $\mathrm{y}$ en posición de prominencia temporal en el discurso. Ambos eventos, con todo, no tienen, desde otro punto de vista, la misma prominencia, pues el evento referido en PA es visto en perspectiva del comunicado en indefinido. La construcción, por tanto, permite, a la vez, poner dos eventos en secuencia temporal y, junto con ello, entrelazarlos fuertemente, poniendo el uno al servicio del otro. Esto es posible porque tanto la cláusula en PA como la en indefinido comunican eventos que hacen avanzar temporalmente el relato. El evento en PA comunica un punto que, en la gran mayoría de los casos, sirve de límite inicial del evento en indefinido, como en (5). Como se verá más abajo, también puede comunicar el punto de término, aunque muy esporádicamente.

\section{EL PRETÉRITO ANTERIOR EN EL QUIJOTE}

Se encontraron 66 casos de PA en El Quijote, dos de ellos en coordinación de dos cláusulas en PA. De los 32 verbos distintos empleados en PA, el más frecuente es ver, con 11 casos, todos en cláusulas transitivas. En todos ellos, ver asume el sentido de reconocer o identificar, como se observa en este ejemplo:

(11) y, apartándose los cabellos de delante de los ojos con entrambas manos, miró los que el ruido hacían; y apenas los hubo visto, cuando se levantó en pie y, sin aguardar a calzarse, ni a recoger los cabellos, asió con mucha presteza un bulto, como de ropa, que junto a sí tenía, y quiso ponerse en huida ( $1^{\mathrm{a}}$ parte, cap. XXVIII). 
Como se advierte, la cláusula en PA comunica el inicio del ver, correspondiente al momento en que el personaje reconoce a quienes hacían ruido. En términos aspectuales, esto significa que el PA coerciona a logro el significado aspectual básico del verbo ver. El verbo ver es, a nuestro juicio, un típico verbo de actividad vendleriana, esto es, un verbo dinámico, durativo y atélico (cfr. Vendler 1967). Diversas pruebas (cfr. Smith 1997) apoyan este análisis: su compatibilidad con la oración Lo que hizo fue ver televisión, restringida a situaciones dinámicas, es decir, no estados; su compatibilidad con cláusulas progresivas como Está viendo televisión en la sala, que exigen situaciones dinámicas durativas (Soto y Castro 2010); la dificultad para interpretar oraciones como Vio televisión en cinco minutos, que suponen realizaciones (situaciones dinámicas, durativas y télicas); y la ausencia de ambigüedad en oraciones como Casi vio televisión, que en el caso de las realizaciones presentan dos interpretaciones posibles (cfr. Casi dibujó el cocodrilo: no empezó a dibujarlo o no terminó de dibujarlo), entre otras pruebas. Al tratarse de un logro derivado por coerción, el significado de ver como reconocer o identificar no es compatible, en sentido propio, con una interpretación progresiva. La interpretación que genera una cláusula como Está viendo a Rodrigo, en el sentido de 'lo está reconociendo', no es que lo reconoce en ese momento sino que está por reconocerlo, que es, precisamente, el significado esperable a partir de la combinación de una cláusula progresiva con un verbo de logro, toda vez que la cláusula, al ser incompatible con logro, coerciona el verbo a actividad (cfr. Esta ganando la carrera, donde ganar es un verbo de logro típico $)^{9}$.

El siguiente ejemplo, tomado de uno de los trozos más conocidos de la obra, muestra nuevamente este empleo de ver como reconocer.

(12) Y queriendo leer otro papel de los que había reservado del fuego, lo estorbó una maravillosa visión (que tal parecía ella) que improvisamente se les ofreció a los ojos; y fue que, por cima de la peña donde se cavaba la sepultura pareció la pastora Marcela, tan hermosa, que pasaba a su fama su hermosura. Los que hasta entonces no la habían visto la miraban con admiración y silencio; y los que ya estaban acostumbrados a verla no quedaron menos suspensos que los que nunca la habían visto. Mas apenas la hubo

$9 \quad$ A diferencia de lo que ocurre con ver, el verbo inglés to see se categoriza como estado (Vendler 1967). Por las pruebas expuestas, discrepamos de la idea de que ver sea estado. Con todo, la idea de que el PA coerciona a logro en el caso de ver es independiente de la caracterización de este último como estado o actividad. 


\section{visto Ambrosio, cuando con muestras de ánimo indignado le dijo:}

- ¿Vienes a ver, por ventura, ¡oh fiero basilisco destas montañas!, si con tu presencia vierten sangre las heridas deste miserable a quien tu crueldad quitó la vida, o vienes a ufanarte en las crueles hazañas de tu condición, o a ver desde esa altura, como otro despiadado Nero, el incendio de su abrasada Roma, o a pisar arrogante este desdichado cadáver, como la ingrata hija al de su padre Tarquino? (1 ${ }^{\mathrm{a}}$ parte, cap. XIV).

En este ejemplo pueden contrastarse dos usos en pluscuamperfecto de ver, claramente de trasfondo, con el empleo en PA, que sigue la secuencia narrativa de primer plano, pero, a la vez, marca el momento mismo en que Ambrosio le habla a Marcela, otorgando gran dinamicidad al segmento. Por supuesto, en este caso el ver, esto es, el reconocer, es condición del decir de Ambrosio. El adverbio cuando, que encabeza la cláusula de decir, es un recurso recurrente en construcciones que contienen una cláusula en PA, aunque no exclusivo de ellas pues puede también aparecer en casos de pluscuamperfecto. Por su carácter puntual o instantáneo, el adverbio cuando refuerza la simultaneidad de los dos eventos, ver y decir. Parece claro que el autor emplea estos recursos para enfatizar el arrebato emocional de Ambrosio.

A ver le sigue andar, con 6 casos. En todos ellos se cuantificó la cantidad andada, de acuerdo con el modelo $X$ anda una cantidad $Y$. Como ver, el verbo andar también es, de suyo, una actividad vendleriana, esto es, un evento dinámico atélico. La incorporación léxica de una cantidad definida impone telicidad en la cláusula comunicada, favoreciendo la coerción a logro desencadenada por PA. También es destacable que en casi todos los casos de andar la cláusula vaya encabezada por no. Veamos algunos ejemplos:

(13) Y no hubieron andado un cuarto de legua, cuando, al cruzar de una senda, vieron venir hacia ellos hasta seis pastores, vestidos con pellicos ( $1^{\mathrm{a}}$ parte, cap. XII).

(14) Y así se fue, aunque todavía le importunaba don Quijote, que le viese siquiera hacer dos locuras. Mas no hubo andado cien pasos, cuando volvió y dijo: -Digo, señor, que vuestra merced ha dicho muy bien ( $1^{\text {a }}$ parte, cap. XXV).

(15) Todas estas razones oyeron y percibieron el Cura y los que con él estaban, y por parecerles, como ello era, que allí junto las decían, 
se levantaron a buscar el dueño, y no hubieron andado veinte pasos, cuando detrás de un peñasco vieron sentado al pie de un fresno a un mozo vestido como labrador ( $1^{\mathrm{a}}$ parte, cap. XXVIII).

La negación opera discursivamente para dar cuenta de que los dos eventos son, prácticamente, simultáneos, lo que se refuerza por el empleo del adverbio cuando. En todos estos casos, el autor enfatiza, además, la rapidez con que ellos ocurren. Puede deducirse que se trata aquí de que un evento, el segundo, es inesperado e irrumpe mientras el otro se despliega. Para decirlo con más precisión, cuando se alcanza un punto particular en el desarrollo de un evento (el punto marcado por la opción de PA), aparece otro contrario a las expectativas generadas en el discurso. Nuevamente, se trata de eventos con una alta dinamicidad - unos más que otros, es cierto. Obsérvese que ya hemos visto que no es compatible con cláusulas que aseveran que el evento ha ocurrido, por lo que no se trata de un operador que marque polaridad negativa. El ejemplo (5) de la caída de don Quijote, que aquí repetimos como (16) en un contexto más amplio, es claro en este sentido:

(16) [Y] no hubo tocado al suelo, cuando se levantó más ligero que un gamo, y comenzó a correr por aquel llano, que no le alcanzara el viento (El Quijote, $1^{\mathrm{a}}$ parte, cap. XXI).

La mayor parte de las cláusulas en PA, 23, comunican, en el corpus, logros correspondientes a eventos de movimiento, típicamente direccionales y télicos, como llegar, apartarse, entrar o salir. También son recurrentes los verbos de percepción como ver, oír y mirar, que en su conjunto suman 17 casos. Si a estos sumamos los verbos puramente cognitivos, el total alcanza los 21 casos. En todos ellos se comunica el cambio que da inicio a cierto estado cognitivo y que sirve de límite a un evento pasado dependiente de ese conocimiento. En 6 oportunidades se trata de verbos de habla, en 5 de ellas un único verbo: decir. También hay 4 instancias con verbos de manipulación, que implican un movimiento télico, como poner o tomar. Asimismo, en 4 oportunidades se trata de eventos físicos transitivos, como comer, hacer, limpiar. Se encuentra un único verbo aspectual, acabar, aunque empleado 3 veces. Hay también unos pocos casos que escapan a estos tipos, pero que siguen siendo dinámicos y télicos, lo que confirma la hipótesis de este trabajo y los hallazgos de la investigación anterior en el español de Chile.

En síntesis, el PA se emplea como un recurso facultativo por parte del autor. Con él, el hablante comunica un logro que sirve de punto de partida a otro evento. Ambos establecen típicamente una secuencia narrativa. $\mathrm{La}$ conceptualización del primer evento como instantáneo o puntual se asocia a lecturas de alto dinamismo. 
En el siguiente ejemplo, las cláusulas en imperfecto establecen el trasfondo de los eventos que, más tarde, harán avanzar el relato. Obsérvese que el evento en PA - un logro vendleriano, llegar- sirve como punto de partida del evento de ir al socorro, lo que lleva a evocar una situación que ocurre con gran rapidez o urgencia:

(17) Sancho estaba tan junto y cosido con él, que casi por línea recta subían los vapores hacia arriba, no se pudo excusar de que algunos no llegasen a sus narices; y apenas hubieron llegado, cuando él fue al socorro, apretándolas entre los dos dedos, y, con tono algo gangoso, dijo:

-Paréceme, Sancho, que tienes mucho miedo (El Quijote, $1^{\mathrm{a}}$ parte, cap. XX).

Por otro lado, también se encuentra en El Quijote un empleo poco usual del PA, que viene del Medioevo y que sigue apareciendo, muy esporádicamente, en el español actual. Este uso, cuya caracterización hemos propuesto en Soto (2009), deja en claro que no siempre el PA comunica una situación anterior a otra pasada al interior de una misma oración. Obsérvese el siguiente caso:

(18) Estas fueron las razones que Anselmo dijo a Lotario, a todas las cuales estuvo tan atento, que si no fueron las que quedan escritas que le dijo, no despegó sus labios hasta que hubo acabado ( $E l$ Quijote, $1^{\mathrm{a}}$ parte, cap. XXXIII).

En casos como este, el PA marca el límite final en que cesa un evento o un estado anterior. Nótese que el estado anterior es negativo y durativo. La primera característica es típica, aunque no obligatoria, de estas construcciones, mientras que la segunda es una restricción obligatoria de la construcción.

\section{COMENTARIOS FINALES}

En el presente trabajo se ha analizado, en un corpus específico, el funcionamiento en la modalidad narrativa de una construcción de muy baja frecuencia en español, mostrando que, con ella, el autor resuelve algunos problemas de organización local del discurso. A pesar de su actual obsolescencia y de su empleo minoritario en todos los estadios del español 
que se han analizado, el PA constituye, en el corpus analizado, un recurso que permite comunicar dos eventos íntimamente ligados y secuencialmente ordenados; marcando, a la vez, que uno de ellos sirve típicamente de punto de partida del otro. Este recurso permite comunicar eventos altamente dinámicos y, mediante este recurso, evocar situaciones de rapidez, violencia o emoción. En los casos en que opera sobre una situación no puntual, el PA coerciona el verbo, generando un logro vendleriano. Los usos de El Quijote ilustran este empleo de modo ejemplar, como es, por lo demás, esperable en un monumento lingüístico como este.

\section{REFERENCIAS BIBLIOGRÁFICAS}

BeLLo, ANDRÉs. 1841/1972. Análisis ideológica de los tiempos de la conjugación castellana. Reproducción facsimilar de la edición príncipe, edición facsimilar. Caracas: Gobernación del Distrito Federal.

Bocaz, Aura. 1997. Las cláusulas relativas y sus funciones en el discurso narrativo infantil. Signo \& Seña 8: 170-186.

Bull, William. 1947. Modern Spanish Verb-form Frequencies. Hispania 30: 451-466.

Criado de Val, Manuel. 1948. Sintaxis del verbo español moderno. Madrid: C.S.I.C. (Anejos de la Revista de Filología Española).

Francis, Elaine y Laura Michaelis. 2003. Mismatch: a crucible for linguistic theory. En Elaine Francis y Laura Michaelis (eds.). Mismatch: form-function incongruity and the architecture of grammar, pp. 1-27. Stanford: CSLI Publications.

GarCíA, Luis. 2008. Pretérito pluscuamperfecto y pretérito anterior. En Ángeles Carrasco (ed.). Tiempos compuestos y formas verbales complejas, pp. 359-400. Madrid: Iberoamericana Vervuet.

Hopper, Paul J. y Sandra A. Thompson. 1980. Transitivity in grammar and discourse. Language 56: 251-299.

Hurtado, Silvia. 2001. El pretérito anterior en castellano medieval. Verba 27: 205-221.

Labov, William. 1997. Some further steps in narrative analysis. Journal of Narrative and Life History 7: 395-415.

Labov, William y Joshua Waletzky. 1967. Narrative analysis. En June Helm (ed.). Essays on the Verbal and Visual Arts, pp. 12-44. Seattle: U. of Washington Press.

Mackenzie, IAn. 1999. Semantics of Spanish verbal categories. Berna: Peter Lang.

Maldonado, Ricardo. 1999. A media voz. Problemas conceptuales del clítico se. México: Universidad Nacional Autónoma de México.

Miranda, Horacio. 1980-1981. Frecuencia de las formas verbales en el habla culta de Santiago de Chile. Boletín de Filología 31: 865-880.

Moreno de Alba, José G. 2006. Valores verbales de los tiempos pasados de indicativo y su evolución. En Concepción Company Company (coord.). Sintaxis histórica de la lengua española. Primera parte, La frase verbal, Vol. 1, pp. 5-94. México: Universidad Nacional Autónoma de México. 
Octavio de Toledo, Álvaro y Javier Rodríguez. 2008. En busca del tiempo perdido: historia y uso de hube cantado. En Ángeles Carrasco (ed.). Tiempos compuestos y formas verbales complejas, pp. 275-357. Madrid: Iberoamericana Vervuet.

Reinhart, Tanya. 1984. Principles of gestalt perception in the temporal organization of narrative text. Linguistics 22: 779-809.

Smith, Carlota. 1997. The parameter of aspect. Segunda edición. Dordrecht: Kluwer Academic Publisher.

Soto, Guillermo. 2009. Vigencia y significado del pretérito anterior. Un estudio a partir del español escrito en Chile. Estudios Filológicos 44: 227-241

2011. Estructura narativa y proyeciones entre situaciones homogéneas y discretas: léxico, gramática y coerción. Lenguas Modernas 37: 109 - 125.

Soto, Guillermo y Daniel Muñoz. 1999-2000. Construcciones medias de alta transitividad en español: un enfoque cognitivo-discursivo. Lenguas Modernas 26-27: 185-208.

Soto, Guillermo y Christian CAStro. 2010. Una caracterización funcional de estar + gerundio como aspecto de fase: progresividad, dinamicidad y lectura de caso (token). RLA, Revista de Lingüistica Teórica y Aplicada 48: 93-113.

TALmy, LeOnard. 2000. Toward a cognitive semantics. 2 vols. Cambridge, MA: MIT Press.

Unger, ChristoPh. 2002. Global coherence, narrative structure, and expectations of relevance. Ponencia presentada en la conferencia "Relevance Theory and Literature", University of Huddersfield, Inglaterra. Disponible en: http://cogprints.org/5440/ [consulta: 30 de enero de 2013].

VendLer, ZeNo. 1967. Verbs and times. Linguistics in philosophy, pp. 97-121. Ithaca: Nueva York, Cornell University Press. 